\title{
Hi Study of the NGC 6744 System
}

\author{
Stuart D. Ryder ${ }^{1}$, Wilfred Walsh ${ }^{2}$ and David Malin ${ }^{3}$ \\ 1 Joint Astronomy Centre, 660 N. A'Ohoku Place, Hilo, HI 96720, USA \\ sryder@jach.hawaii.edu \\ 2 Radioastronomisches Institut der Universität Bonn, Auf dem Hügel 71, \\ Bonn D-53121, Germany \\ wwalsh@astro.uni-bonn.de \\ 3 Anglo-Australian Observatory, PO Box 296, Epping, NSW 1710, Australia \\ dfm@aaoepp.aao.gov.au \\ Received 1998 November 2, accepted 1999 February 2
}

\begin{abstract}
We present the preliminary results of our 5-configuration, 20-pointing mosaic with the Australia Telescope Compact Array of the neutral hydrogen in the nearby spiral galaxy NGC 6744. The bulk of the Hi resides in a 'ring' underlying the outer optical disk, with $2 \mathrm{HI}$ spiral arms extending further out to almost 1.5 optical radii. The velocity field is fairly regular, apart from evidence for streaming motions along the $\mathrm{H}_{\mathrm{i}}$ arms, and the influence of the companion IB(s)m galaxy NGC $6744 \mathrm{~A}$. We associate a cloud of $\mathrm{H}_{\mathrm{I}}$ at a heliocentric velocity of $846 \mathrm{~km} \mathrm{~s}^{-1}$ with another companion object, ESO 104-g44. Our attempts to construct a mass model for NGC 6744 suffer from poor resolution in the inner disk, and the uncertainty in the total Hi flux of NGC 6744. We anticipate that HIPASS observations will be crucial in resolving the latter issue.
\end{abstract}

Keywords: galaxies: individual (NGC 6744) — galaxies: interactions - galaxies: kinematics and dynamics — galaxies: spiral

\section{Introduction}

NGC 6744 is one of the largest, nearest $(D \sim 11$ $\mathrm{Mpc}$ ), and most spectacular spiral galaxies in the southern sky, and yet it has received surprisingly little attention. In part, this can be attributed to its large angular size (the $B=25 \mathrm{mag} \operatorname{arcsec}^{-2}$ isophote spanning some $\left.20^{\prime} \times 13^{\prime}\right)$ which, besides yielding a relatively low surface brightness disk, exceeds the field of view of most existing optical and radio imaging systems. The recent advent of large format optical CCD arrays, and a mosaic observing mode with the Australia Telescope Compact Array (ATCA) have at last made it feasible to carry out a full investigation of the stellar and gaseous components of NGC 6744. This paper outlines some preliminary results of our Hi observations.

Our study of NGC 6744 was primarily motivated by a desire to understand the influence of the spiral density wave pattern on star formation. NGC 6744 is morphologically very similar to our Galaxy (de Vaucouleurs 1983), but is unusually isolated, with no nearby companion akin to M31. However, deep optical images suggest also some form of interaction between NGC 6744 and the small blue irregular galaxy NGC 6744A, and possibly with a host of other dwarf galaxies in the vicinity. In addition, NGC 6744 is listed in the Catalog of Southern Ringed Galaxies (Buta 1995) as having an inner ring 3.3 in diameter. As has been shown in our earlier ATCA Hi study of the ringed barred spirals NGC 1433 and NGC 6300 (Ryder et al. 1996) that associating such ring features with specific orbital resonances (in this case, the inner second harmonic resonance) is a powerful technique for measuring directly such fundamental dynamical parameters as the bar pattern speed.

\section{ATCA Observations}

NGC 6744 was observed with a total of five different configurations of the ATCA in the period between April and November of 1996. Further details of the observations are given in Table 1 . To be sure of covering the entire Hi disk of NGC 6744 and its nearest neighbours, we mapped a 20 pointing mosaic covering almost $120^{\prime} \times 100^{\prime}$, as indicated in Figure 1 . The calibrator PKS B1934-638 served as both the primary and secondary flux calibrator, on account of its close proximity $\left(3 \cdot 3^{\circ}\right)$ to NGC 6744 . The data for each configuration were edited and calibrated with AIPS. Mosaicing, imaging, and deconvolution were carried out using the MIRIAD suite of software (Sault, Teuben \& Wright 1995), which implements the 'joint approach' to interferometric mosaicing discussed in Sault, Staveley-Smith \& Brouw (1996).

A maximum entropy-based deconvolution task was used to deconvolve the mosaiced dirty image. While being well-suited to imaging of extended objects, the algorithm leads to an inherently positive bias in the 
flux. This bias can be minimised if the total source flux is known a priori, but of course, one of the reasons for doing a mosaic of NGC 6744 in the first place was to determine the total extent and mass of HI. Since the total flux is not yet well-constrained, each of the channel maps in the cleaned Hi cube exhibits a 'pedestal' of emission underneath the dominant gaseous component at each velocity. For this reason, we confine the present analysis mainly to the kinematical aspects of NGC 6744, which are less affected by this positive bias.

Table 1. ATCA observing parameters for NGC 6744

\begin{tabular}{lc}
\hline \multicolumn{1}{c}{ Parameter } & Value \\
\hline$\alpha$ (J2000 pointing centre) & $19^{\mathrm{h}} 09^{\mathrm{m}} 45.3$ \\
$\delta$ (J2000 pointing centre) & $-64^{\mathrm{s}} 01^{\prime} 21^{\prime \prime}$ \\
Distance adopted & $10 \cdot 4 \mathrm{Mpc}$ \\
Configurations $^{A}$ & $0 \cdot 375,0 \cdot 75 \mathrm{~A}, 0 \cdot 75 \mathrm{D}$, \\
& $1 \cdot 5 \mathrm{~A}, 1 \cdot 5 \mathrm{D}$ \\
Natural-weighted beam FWHP & $54^{\prime \prime} \times 51^{\prime \prime}(2 \cdot 7 \times 2 \cdot 6 \mathrm{kpc})$ \\
Channel map rms noise & $3 \cdot 6 \mathrm{mJy} \mathrm{beam}^{-1}$ \\
Channel increment & $9 \cdot 9 \mathrm{~km} \mathrm{~s}^{-1}$ \\
\hline
\end{tabular}

$\overline{\mathrm{A}}$ For further details about the configurations, consult the ATCA Users' Guide.

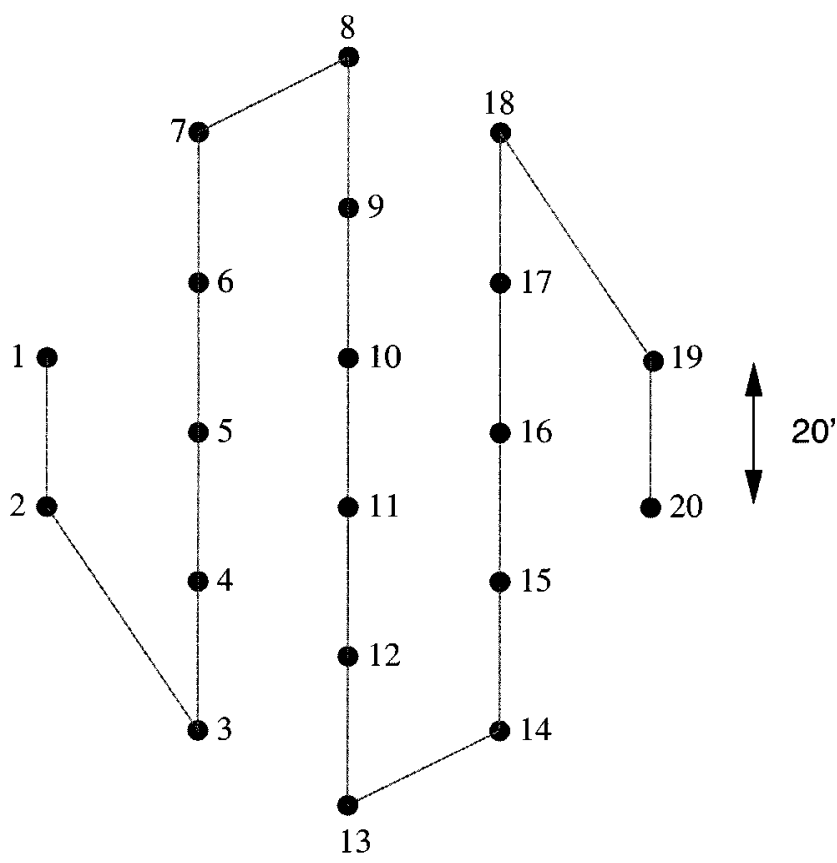

Figure 1-Mosaic pattern on the sky for NGC 6744, as traced by the ATCA antennas. Integrations of $3 \times 10 \mathrm{~s}$ were averaged before moving on to the next position.

\section{Results}

In Figure 2, we have superimposed contours of the Hi column density on an optical image of NGC 6744 extracted from the Digitized Sky Survey. As can be seen, the gas disk extends over some $30^{\prime}$, or 1.5 times the size of the stellar disk. A 'ring' of gas underlies the optical spiral arms, with comparatively little $\mathrm{HI}$ in the bulge and inner disk. Two gaseous spiral arms emanate from either end of this ring, and wind for almost $270^{\circ}$ each before fading out. The peak gas column densities occur where these arms join the ring, and near the location of NGC $6744 \mathrm{~A}$, the irregular galaxy visible to the NW.

A better impression of the structure of the $\mathrm{HI}$ disk, together with its velocity field, can be had from Figure 2. The rotation pattern is basically that expected for gas in uniform circular motion, but distortions are evident, particularly in the gaseous spiral arms and around NGC 6744A. By examining the $\mathrm{HI}$ datacube, we have been able to kinematically resolve NGC $6744 \mathrm{~A}$ from the $\mathrm{HI}$ arm behind it, and find a redshift for this galaxy of $765 \pm 10 \mathrm{~km} \mathrm{~s}^{-1}$. As Figure 3 shows, we have also detected a cloud of $\mathrm{HI}$ to the SE. According to the NASA/IPAC Extragalactic Database, this cloud coincides with the location of the SABm galaxy ESO 104-g44. However, we observe Hi line-of-sight velocities in this cloud of $846 \pm 3 \mathrm{~km} \mathrm{~s}^{-1}$ (with virtually no gradient), in contrast with the $\mathrm{HI}_{\mathrm{I}}$ heliocentric velocity of 779 $\mathrm{km} \mathrm{s}^{-1}$ measured by Longmore et al. (1982) using the Parkes $64 \mathrm{~m}$ antenna. The difference is most likely due to spillover into the beam from blue-shifted gas in the disk of NGC 6744 .

\section{Rotation Curve and Dark Matter Model}

Using both the VELFIT task within MIRIAD and the GAL task within AIPS, we have attempted to model the velocity field of Figure 3. By fixing only the rotation centre, we find a best-fitting systemic velocity $V_{\text {sys }}=838 \pm 2 \mathrm{~km} \mathrm{~s}^{-1}$, an inclination $i=(50 \pm 4)^{\circ}$, and a line-of-nodes position angle $\theta=(16 \pm 2)^{\circ}$. Fixing all of these parameters then yields the rotation curve in Figure 4, where the error bars reflect the differences from analysing separately the receding and approaching halves of the disk. As can be seen, the rotational velocity rises fairly quickly within the first $3 \mathrm{kpc}$ (due to beam smearing, the actual rise is probably steeper), and then stays just below $200 \mathrm{~km} \mathrm{~s}^{-1}$ over the rest of the disk. There is the hint of a rise in the last few points, but the analysis is complicated here by the influence of NGC $6744 \mathrm{~A}$, and apparent streaming motions along the arms.

In order to derive the contribution of a dark matter halo to the rotation curve of NGC 6744 , we must first compute the contributions of the stellar and gaseous components. Using a $2 \mathrm{~K} \times 2 \mathrm{~K} \mathrm{CCD}$ on the Mount Stromlo and Siding Spring Observatories' $40^{\prime \prime}$ reflector, we have constructed a $B$-band mosaic of NGC 6744 covering some $35^{\prime} \times 37^{\prime}$, which has allowed us to determine the run of mean surface brightness with radius in the disk. As a starting point, we assume $M / L_{B}=1$ (although this is almost certainly a lower limit) which then gives the radial distribution of stellar surface density. We derive the radial atomic gas mass profile by scaling the 


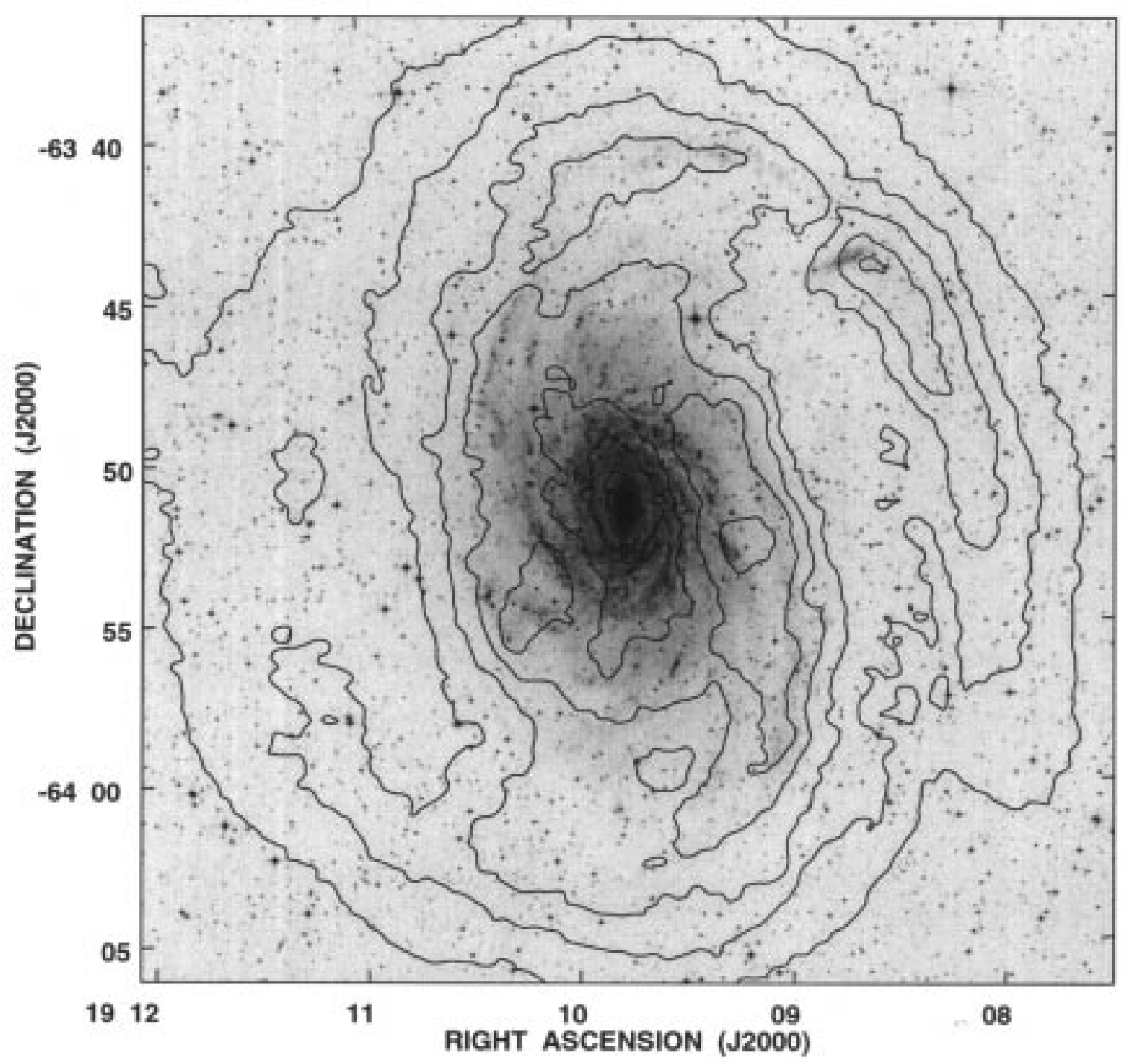

Figure 2 - Contours of the Hi column density (resolution $\sim 55^{\prime \prime}$ ) overlaid on a blue image of NGC 6744 from the Digitized Sky Survey. The IB(s)m galaxy NGC $6744 \mathrm{~A}$ can be seen near $\left(19^{\mathrm{h}} 09^{\mathrm{m}},-63^{\circ} 44^{\prime}\right)$.

Hi surface density by $4 / 3$ (to account for He). The relative contributions of the gas, stars, and dark matter to the rotation curve of NGC 6744 have been computed (assuming that the stellar disk dominates in the very inner disk), and the results are shown by the various curves in Figure 4 .

There are a number of points to note about this mass model. First, the model curve cannot account for all of the structure in the observed rotation curve, and has particular difficulty in the central $10 \mathrm{kpc}$. Also note that for the first $20 \mathrm{kpc}$ or so, the rotation velocity of the gas is negative. This comes about as a result of the concentration of $\mathrm{HI}$ in the ring producing a net outward force, so that the balance of gravitational and centripetal forces requires that the square of the circular velocity $V_{\mathrm{c}}^{2}$ be negative. Therefore $V_{\mathrm{c}}$ must be imaginary, but by convention is plotted as the negative of the real part. The implied isothermal dark halo core radius is $1.83 \mathrm{kpc}$, which is low but not unreasonable, while the halo central density is $2 \cdot 10 \times 10^{-3} M_{\odot} \mathrm{pc}^{-3}$. This mass model must be considered preliminary until we can better constrain the total Hi mass in NGC 6744, and measure the inner rotation curve at higher resolution.

\section{Future Work}

Because the Hi in NGC 6744 overfills the Parkes beam, measuring the total $\mathrm{Hi}$ flux requires either an elaborate mapping strategy, or a smaller antenna. However, we are optimistic that with appropriate processing, the multiple HIPASS scans of the environment of NGC 6744 will enable us to extract a total Hi flux that will serve to constrain the maximum-entropy deconvolution. We have recently acquired $\mathrm{H} \alpha$ FabryPerot scans of the inner disk of NGC 6744; as the images by Ryder \& Dopita (1993) indicate, there is diffuse $\mathrm{H} \alpha$ emission interior to the ring which should enable us to trace ionised gas motions in the bar region. In addition, we plan to map the $\mathrm{CO}$ 


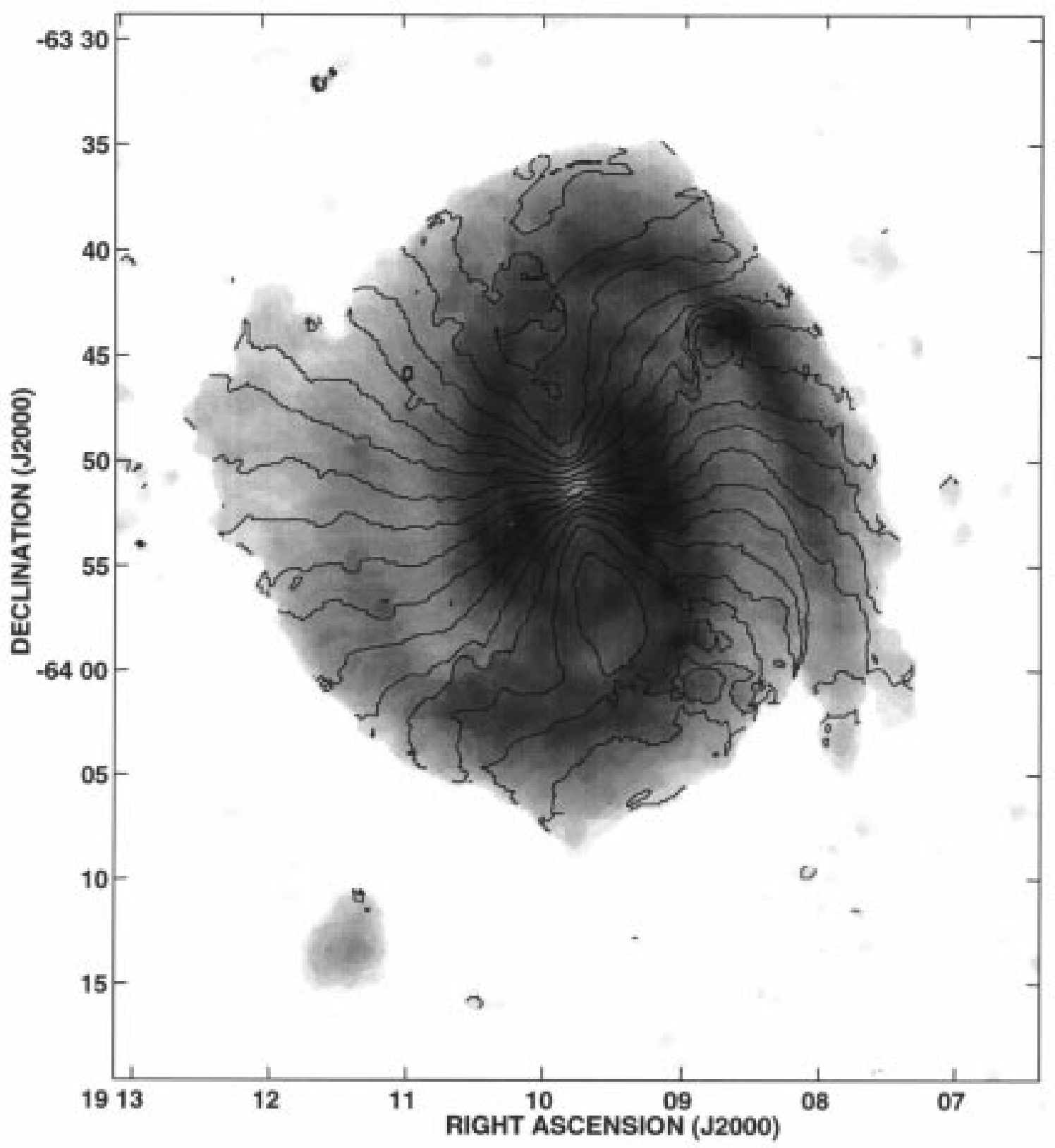

Figure 3-Isovelocity contours for the Hi in NGC 6744, overlaid on a grey-scale of the same Hi distribution as in Figure 2. Notice the severe distortion in the contours due to NGC 6744A. The isolated patch of HI to the SE is associated with ESO $104-\mathrm{g} 44$.

emission from the inner disk of NGC 6744 with the Swedish-ESO Sub-millimetre Telescope. Together, these complementary observations should enable us to understand the internal kinematics of NGC 6744, just as our ATCA mosaic of the Hi has begun to teach us about the large-scale dynamics of the NGC 6744 system.

\section{Acknowledgments}

We are grateful to the Australia Telescope Time Allocation Committee for granting us all the observing time necessary to complete this project. Part of this work was supported by the award of a
UNSW Vice-Chancellor's Postdoctoral Fellowship to SDR. This research has made use of the NASA/IPAC Extragalactic Database (NED) which is operated by the Jet Propulsion Laboratory, California Institute of Technology, under contract with the National Aeronautics and Space Administration. The Digitized Sky Surveys were produced at the Space Telescope Science Institute under US Government grant NAG W-2166. The images of these surveys are based on photographic data obtained using the Oschin Schmidt Telescope on Palomar Mountain and the UK Schmidt Telescope. The plates were processed into the present compressed digital form with the permission of those institutions. 


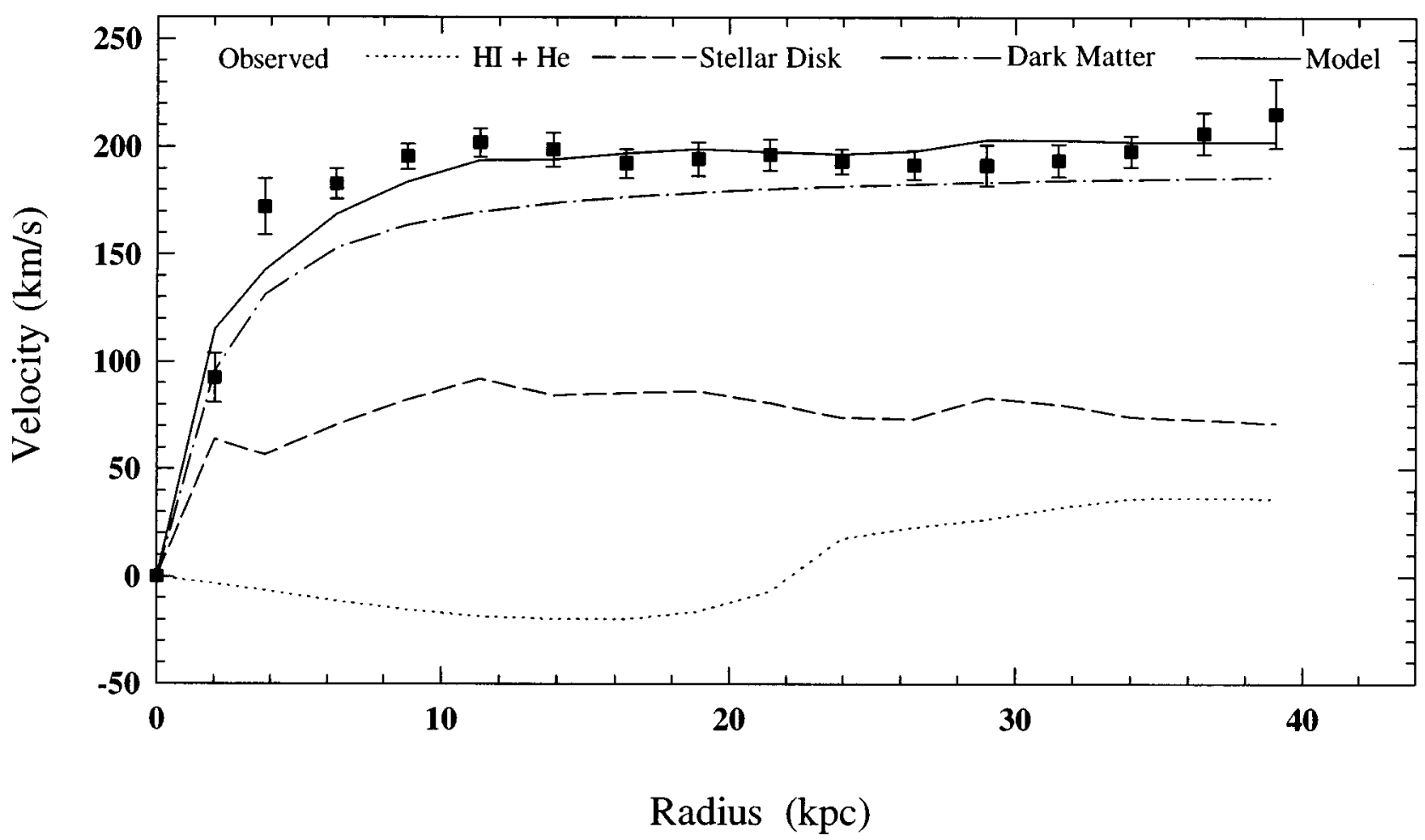

Figure 4-Rotation curve for NGC 6744 , derived from a kinematical analysis of the Hi disk. The points with error bars correspond to the range of values found by analysing both the entire disk, and the receding and approaching halves separately. The dotted curve indicates the rotation due to the atomic gas only, the dashed curve indicates the rotation due to the stellar disk, the dash-dot curve is rotation due to the inferred dark matter component, and the solid curve is the sum of all three components.

\section{References}

Buta, R. J. 1995, ApJS, 96, 39

de Vaucouleurs, G. 1983, ApJ, 268, 468

Longmore, A. J., Hawarden, T. G., Goss, W. M., Mebold, U., \& Webster, B. L. 1982, MNRAS, 200, 325

Ryder, S. D., \& Dopita, M. A. 1993, ApJS, 88, 415

Ryder, S. D., Buta, R., Toledo, H., Shukla, H., Staveley-

Smith, L., \& Walsh, W. 1996, ApJ, 460, 665
Sault, R. J., Staveley-Smith, L., \& Brouw, W. N. 1996, A\&AS, 120, 375

Sault, R. J., Teuben, P. J., \& Wright M. C. H. 1995, in Astronomical Data Analysis Software and Systems IV, ASP Conf. Ser. Vol. 77, ed. R. A. Shaw et al. (San Francisco: ASP), p. 433 